\title{
The use of local features and global spatial context for object recognition in a visuomotor task in the Mongolian gerbil
}

\author{
COLIN G. ELLARD and MARLA G. BIGEL \\ University of Waterloo, Waterloo, Ontario, Canada
}

\begin{abstract}
Mongolian gerbils were trained to jump across gaps of randomly varying width in order to obtain a food reward. During training, gerbils learned to jump to each of two landing platforms differing in width. These landing platforms were associated with unique spatial contexts (Experiment 1), local features (Experiment 2), or both (Experiment 3). In test sessions, a landing platform was substituted that was intermediate in width between the two training platforms of novel width, in order to determine whether gerbils could use retinal image size to calibrate distance. Experiments 1 and 2 suggested that gerbils used spatial context but not local feature information to identify the target platforms. In Experiment 3 , when the probe platform was presented with the same local feature information and in the same context as seen in training sessions, gerbils over- or underjumped in a manner predicted if they were using the retinal image size of the target to calibrate the distance across the gap. When the probe trials contained a mismatch between context and local feature information, no systematic over- or underjumps were seen, and jumping accuracy was decreased. Taken together, these results suggest that, in this task, objects are identified primarily on the basis of the spatial context in which they are seen and not on the basis of their local features.
\end{abstract}

In previous experiments, we have shown that gerbils are able to calibrate precisely the distance across a gap in order to jump across that gap to obtain a reward (see Ellard \& Goodale, 1991, for review). Among the depth algorithms that gerbils use to generate estimates of target distance is retinal image size (RIS). Because there is a systematic relationship between the distance of a target from the eye and the size of its retinal image, gerbils that are trained to jump to a target of particular dimensions will produce predictable errors if a probe platform is introduced that differs in width from the training platform (Goodale, Ellard, \& Booth, 1990). Wider probe platforms produce underjumps, and narrower probes cause the gerbils to overjump.

Unlike many other depth algorithms, RIS can only provide distance information if the animal has learned a calibration of image size based on the real dimensions of the target. In humans, for instance, illusions of distance based on RIS are most compelling when highly familiar objects, such as coins, are used as the targets (Epstein \& Baratz, 1964). In our previous studies with gerbils, we have attempted to optimize the use of RIS by overtraining gerbils to jump to a platform whose dimensions are fixed. In this situation, RIS is a reliable cue to distance.

This research was supported by a grant to C.G.E. from the Natural Sciences and Engineering Research Council of Canada. The authors wish to thank David Carey for valuable criticisms of an earlier version of this study and Katherine Anderson and Myra Fernandes for their careful collection of some of the data. Correspondence should be addressed to C. G. Ellard, Department of Psychology, University of Waterloo, Waterloo, ON, Canada N2L 3G1.
If RIS is to be a useful depth cue for gerbils, it must be possible for them to calibrate the relationship between image size and object distance for a number of different objects and to recall these calibrations in the appropriate circumstances. In other words, RIS would be most useful if gerbils were able to compile a "catalog" of veridical object dimensions and to use the appropriate entry in the catalog in the appropriate circumstances. In a recent experiment (Ellard, Chapman, \& Cameron, 1991), we demonstrated that gerbils can store and recall two separate calibrations of objects when each of the calibrations is associated with a particular spatial context. In that experiment, gerbils were trained to jump in two distinct contexts (two different locations within the lab); in each context, they were presented with a landing platform of set dimensions (a wide platform in one room and a narrow platform in the other). On probe trials, gerbils were presented with a platform that was intermediate in width between the narrower and the wider of the two platforms. The outcome of the experiment was that gerbils either over- or underjumped to the probe platform, depending only upon the context in which they were presented with the probe platform (and, consequently, whether they were expecting a narrow or wide platform).

In this experiment, it was important that the identical probe platform was used in both contexts in order to rule out the possibility that the results were produced by differences in the visual features of the landing platforms. Therefore, we do not know whether local feature information can be used to identify targets for the purpose of RIS calibration. This is an important question because accumulating anatomical, physiological, and neuropsycho- 
logical evidence suggests that rodents are adapted to place strong precedence on spatial information rather than local feature information, even for the purpose of identifying objects (Ellard \& Carey, 1996).

In the present experiments, we manipulated spatial context and local feature information separately in order to measure their relative importance for retinal image size calibration. In the first experiment, gerbils were trained to associate a particular target size with a spatial context by training in two separate rooms. In the second experiment, we trained gerbils to associate a target size with a set of local features - a distinctive pattern on the face of each of the two target platforms. In the third experiment, we trained gerbils to jump to platforms that were associated with both a distinct set of local features and a particular spatial context. In testing sessions, these two sources of information about the identity of the target platform were thrown into conflict in order to assess the relative salience of local and global information on identity judgments.

\section{EXPERIMENT 1}

Experiment 1 was a replication of Ellard et al. (1991). Gerbils were trained to jump to two different standard widths of platform in two different spatial contexts. In testing sessions, gerbils were presented with an intermediate-sized platform. If they were able to associate two separate spatial contexts with two different objects, then presentation of the probe platform ought to produce predictable over- or underjumps. The use of the identical probe platform in both spatial contexts during training is a strong test of the use of RIS, since it guards against the possibility that performance is being affected by some peculiarity of the platform itself.

\section{Method}

Subjects. Five male Mongolian gerbils (Meriones unguiculatus) ranging in age from 4 to 8 months at the beginning of training were used. Each gerbil was maintained at approximately $90 \%$ of its freefeeding weight by feeding measured amounts of Purina lab chow once every $24 \mathrm{~h}$. They were also provided with ad-lib access to water. One day a week, each gerbil was given a small portion of a dietary protein supplement (Purina calf starter). The gerbils were housed individually in Plexiglas cages and were kept on a 12:12-h light:dark schedule. The gerbils were always tested during the light phase.

Apparatus. The jumping apparatus consisted of a takeoff platform $(20 \mathrm{~cm}$ wide $\times 21 \mathrm{~cm}$ high $\times 32 \mathrm{~cm}$ long; the leading edge of the takeoff platform tapered off to a width of $6 \mathrm{~cm}$ ) and two landing platforms - a narrow platform $(11 \mathrm{~cm}$ wide $\times 31 \mathrm{~cm}$ long $\times$ $21 \mathrm{~cm}$ high) and a wide platform $(26 \mathrm{~cm}$ wide $\times 31 \mathrm{~cm}$ long $\times$ $21 \mathrm{~cm}$ high). The surfaces of both the takeoff and the landing platforms were covered with black textured vinyl, which provided the gerbils with traction during takeoff and landing. Two different rooms in the laboratory were used for training and testing (Room $A$ and Room B). The platforms rested on a table in each testing room, and a background was placed on the wall $80 \mathrm{~cm}$ behind the landing platform in both rooms to provide additional contextual information. The background in Room A consisted of randomly distributed lines varying in length, orientation, and thickness; the background in Room B consisted of randomly distributed filled black circles that varied in size.
Training. The animals were divided into two groups. Group 1 $(n=3)$ received training with the narrow platform in Room $\mathrm{A}$ and training with the wide platform in Room B. Group $2(n=2)$ received complementary training (wide platform in Room A, narrow platform in Room B). Initially, the takeoff and landing platforms were placed in contact with one another. A morsel of food was placed in the center of the landing platform, and each gerbil was left to explore the apparatus for $5 \mathrm{~min}$ a day until it would reliably travel to the landing platform and eat the food. Next, a small gap was introduced between the two platforms. Daily training consisted of 12 trials for each animal: 6 trials in Room A and 6 trials in Room B, in accordance with their group designations. The trials were conducted in random order. Over the course of training, the gap width was slowly increased until all the gerbils could jump a gap distance of $30 \mathrm{~cm}$ successfully and consistently. Training took approximately 2 months, and the gerbils were deemed ready for testing when they could successfully complete a jump within $1 \mathrm{~min}$ of being placed onto the takeoff platform.

Testing. Testing took place over 4 days. Each test session consisted of 16 trials: 8 trials in Room A and 8 trials in Room B. The testing protocol was organized such that the gap distances and the room order were varied randomly each session. Gap distances ranged from 10 to $28 \mathrm{~cm}$, in $6-\mathrm{cm}$ increments. During each testing session, the gerbils received 2 probe trials that were randomly interspersed among the control trials. The probe trials consisted of presenting a platform of intermediate width $(15.5 \mathrm{~cm}$ wide $X$ $21 \mathrm{~cm}$ high $\times 31 \mathrm{~cm}$ long) in each room at one of four gap distances: $10,16,22$, or $28 \mathrm{~cm}$.

Analysis. The gerbils were videotaped using a Panasonic 190 VHS movie camera for recording and playback. Single fields from the videotape record were saved on a Macintosh computer using a frame-grabbing interface (Videospigot, SuperMac Technologies). From the image, we measured the horizontal and vertical coordinates of the leading edge of both the takeoff and the landing platforms, as well as the horizontal and vertical coodinates of the forepaw touchdown location. In cases where the gerbils jumped short of the landing platform, touchdown location was measured at the point at which the forepaw crossed the horizontal plane that connected the takeoff and landing platforms. These coordinates were converted to distances by calibrating a known distance in the image.

\section{Results}

Figure 1 shows the effects of presenting the probe platform in different spatial contexts (i.e., in either Room A or Room B) at each of the four gap distances. As can be seen from the figure, the effect of presenting the probe platform in the spatial context where the animal had been trained to jump to a wide platform produced overjumps relative to control performance at gap distances of 16 , 22 , and $28 \mathrm{~cm}$. When the animals were presented with the probe platform in the spatial context where they had been trained to jump to a narrow platform, the effect was to produce an underjump relative to control performance at all gap distances.

Results of a repeated measures analysis of variance (ANOVA) showed that there was a marginally significant effect of probe type $[F(2,8)=4.05, p<.1]$, but there was no significant interaction between probe type and gap distance $[F(6,24)=1.87, p<.1]$. There was, however, a significant main effect of gap distance $[F(3,12)=$ $12.11, p<.05]$, and a Newman-Keuls test of means revealed that the animals' landing positions on the platforms at gap distances of 16 and $22 \mathrm{~cm}$ differed significantly 


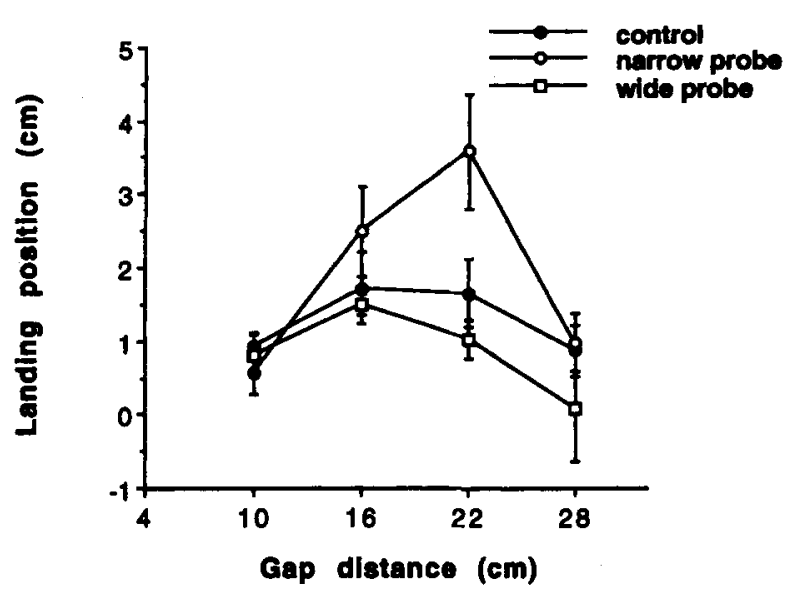

Figure 1. Line graph showing mean landing position as a function of gap distance for probe and control trials in Experiment 1. Solid circles represent performance on control trials. Open circles represent performance on those trials in which the gerbils would expect narrow training platforms based on training conditions. Open squares represent performance on those trials in which the wide training platform would be expected. Performance is averaged over all animals. Error bars represent standard errors of the mean.

from the gap distances of 10 and $28 \mathrm{~cm}$ but not from each other [Newman-Keuls, $F(3,56)=6.71, p<.05]$. To measure trial-to-trial consistency in performance, we computed a variance measure for individual gerbils in each of the different types of conditions in the experiment. To obtain sufficient numbers of individual trials to compute error scores for individual animals, we lumped across trials at different distances. For each gerbil, we computed the standard deviation $(S D)$ in landing position on control trials and on probe trials. Because average landing positions on the narrow-expected and wide-expected probe trials differed, we computed two separate $S D$ scores for each of the probe types and averaged them. Hence, the data for analysis consisted of two scores for each animal: an error score for the control condition, and one score for probe conditions. Results of a correlated $t$ test showed that there was a significant trend for increased error on probe trials relative to control trials $[t(4)=2.53$, $p<.1]$.

\section{Discussion}

In accord with the findings of Ellard et al. (1991), the results of this experiment suggest that when gerbils are trained to jump to two different targets in two spatial locations, they can learn separate calibrations between the apparent image size of the two targets and their distance. This form of testing for the use of RIS allows the strongest claims regarding the explanation for the over- and underjumps produced on probe trials. Although it is not likely, one could argue that subtle differences between the probe platforms and the training platform might have been responsible for part of the probe effect reported by Goodale et al. (1990). In our earlier work (Ellard et al., 1991) and in the present study, this confound was removed by hav- ing the gerbils jump to the same probe platform in two different contexts, where their expectations were based on the width of training platform that was associated with that context. The presence of either under- or overjumps to the same probe platform by the same animal, depending only upon context, rules out these alternative explanations for the probe effect.

Although there are some suggestions of such a trend in earlier work (Ellard et al., 1991; Goodale et al., 1990), our observation is novel that the size of an animal's overjump appears to vary with gap distance. The simplest explanation for this is that, at short distances, gerbils are able to compute distance with sufficient accuracy that little margin for error is required and, at long distances, performance is limited by the jumping abilities of the animals. In other studies, though, gerbils have jumped distances of up to $40 \mathrm{~cm}$ (Ellard, Goodale, \& Timney, 1984; Ellard \& Ilkov-Moor, 1995). Another more interesting but quite speculative explanation for the variability in performance with gap distance is that feedback from the results of probe trials has rendered estimates of distance from RIS relatively more unreliable (Ellard \& Goodale, 1991), and, so, in the absence of compensatory sources of depth information at intermediate distances (Collett \& Harkness, 1981), gerbils jump conservatively at middle distances.

The finding of significant increases in variability in landing position on probe trials relative to control trials suggests that gerbils are generating less consistent estimates of distance on the probe trials. The misleading RIS information on these trials, provided that it was being used in the same way as veridical RIS information, should not decrease accuracy. Rather, these decreases in accuracy suggest that less information is being used on probe trials and, logically, it seems most likely that it is the spurious information from RIS that is being discounted. One possible reason for this is that gerbils are noticing that the probe platform is not the same as the training platforms, and they either are disregarding RIS or are weighting it less heavily.

In conclusion, we were able to replicate the main finding in Ellard et al. (1991) that, for the purpose of retrieving RIS calibrations, gerbils could distinguish between objects on the basis of spatial location, even when no local feature information was present.

\section{EXPERIMENT 2}

In Experiment 1, we demonstrated that gerbils could use spatial context to identify the target platform for the purpose of retrieving a calibration of the distance of the target with its RIS. As in previous experiments, we did not provide local feature information to the gerbils that might have been used to indicate which of the two target platforms was being presented. Although there were sound reasons for withholding this information in previous work, this kind of procedure is somewhat at odds with the human psychophysical literature, for instance, on the uses of RIS. In those studies (Epstein \& Baratz, 1962, for instance), 
highly familiar objects are used whose familiarity is based upon local features rather than upon spatial context. In Experiment 2, we were interested in finding out whether gerbils could use local feature information for RIS calibration. In Experiment 2, all testing was conducted in the same spatial context, but the two training platforms were made distinctive by the addition of conspicuous local features.

\section{Method}

Subjects. Seven male Mongolian gerbils (Meriones unguiculatus) ranging in age from 4 to 8 months at the beginning of training were used. Housing and maintenance schedules were the same as those used in Experiment 1.

Apparatus. The jumping apparatus was identical to that used in Experiment 1, except that local features were added to the leading faces of the landing platforms using 1-cm-wide white tape. One side of each of the two landing platforms was covered with oblique stripes (spaced $1 \mathrm{~cm}$ apart), and the other side was covered with a random arrangement of $1-\mathrm{cm}$ square white patches. The background behind the landing platform consisted of randomly distributed filled black circles that varied in size. All other room conditions remained the same as those in Experiment 1.

Training. The gerbils were divided into two groups. Group 1 $(n=3)$ was trained to jump to a wide platform with stripes and a narrow platform with dots. Group $2(n=4)$ was trained to jump to a narrow platform with stripes and a wide platform with dots. All training took place in the same room. The gerbils were trained to jump across a gap using the same methods as those in Experiment 1. After the gerbils became accustomed to the apparatus, the gap sizes and local features were varied randomly each day so that the animals could not predict the order in which the platforms were presented. Care was taken to ensure that the animals in each group only saw each of the two platform widths with one type of local feature, according to group assignment. The gerbils were deemed ready for testing if they successfully completed a jump within $1 \mathrm{~min}$ of being placed on the takeoff platform, and they jumped reliably and accurately across a gap of at least $30 \mathrm{~cm}$. Training for this experiment took approximately $2-3$ months.

Testing. The testing procedure in this experiment was the same as that for Experiment 1, except that local features were varied rather than spatial context. Testing took place over 4 days. Each test session consisted of 16 trials: 8 trials with a wide platform and 8 trials with a narrow platform. Each group was tested with the appropriate pattern paired with each width of platform. The gap distances and the platform patterns were varied randomly each session. Gap distances ranged from 10 to $28 \mathrm{~cm}$. During each testing session, the gerbils received 2 probe trials that were randomly interspersed among the control trials. In the probe trials, the gerbils jumped to a platform of intermediate width $(15.5 \mathrm{~cm}$ wide $\times 21 \mathrm{~cm}$ high $\times$ $31 \mathrm{~cm}$ long), and they saw each of the two types of local features. Over the four testing sessions, the probe trials were at one of four gap distances: $10,16,22$, or $28 \mathrm{~cm}$.

\section{Results}

Figure 2 shows the effects of presenting the probe platform (with either dots or stripes as local features) at each of the four gap distances. As can be seen from the figure, there was no consistent effect of probe type on landing position in this experiment.

A repeated measures ANOVA was conducted to analyze the data. Results of the ANOVA showed that there was no significant probe effect $[F(2,12)=1.22, p>.1]$. As in Experiment 1, there was a main effect of distance $[F(3,12)=12.11, p<.05]$, but no significant interaction

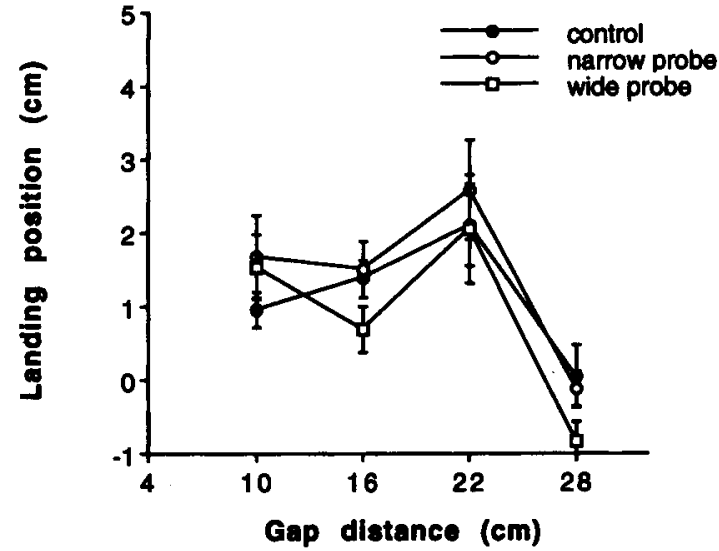

Figure 2. Line graph showing mean landing position as a function of gap distance for probe and control trials in Experiment 2. Solid circles represent performance on control trials. Open circles represent performance on those trials in which the narrow training platform would be expected. Open squares represent performance on those trials in which the wide training platform would be expected. Performance is averaged over all animals. Error bars represent standard errors of the mean.

was noted between probe type and gap distance $[F(6,24)=$ $0.67, p>.1]$. The gerbils overjumped consistently less, regardless of the probe's local features, at a gap distance of $28 \mathrm{~cm}$ and overjumped the most at a gap distance of $22 \mathrm{~cm}$. Analysis of variability in landing position showed no differences between probe and control trials $[t(6)=$ $1.56, p>.1]$.

\section{Discussion}

In Experiment 2, we predicted that since there was no change in global spatial context, the gerbils would use information about the local features of the landing platforms to identify the platforms and, hence, retrieve the correct RIS calibration from memory. However, we found that altering the appearance of the landing platforms by placing either stripes or dots onto their faces did not provide enough information for the gerbils to retrieve the correct calibrations. There are two possible explanations for the failure to use RIS: either the gerbils could not see the local features on the landing platforms or they were not able to use them. With regard to the first explanation, Baker and Emerson (1983) have estimated the squarewave grating acuity of the gerbil as $1.5-2$ cycles per degree (cpd). Even at the longest distances tested, the local features that we used would have had a spatial frequency of approximately $0.5 \mathrm{cpd}$, well within these limits. Hence, the local features on the platforms would have been easily visible to the gerbils, and, in other kinds of tasks, gerbils have been able to solve discrimination problems employing similar types of stimuli (Ingle, 1981). These data suggest that, in our task, gerbils do not use local features of the landing platforms alone to identify objects for the purpose of RIS calibration.

Just as in Experiment 1, the gerbils in Experiment 2 tended to jump consistently farther from the leading edge of the landing platform at intermediate gap distances. 
Previously, we suggested that overjumps were greatest at these distances because of the relative unavailability of independent distance information at these distances relative to that available at shorter and longer distances.

\section{EXPERIMENT 3}

In Experiment 2, we showed that, in contrast to spatial context information, local feature information is not used by gerbils to distinguish between two target platforms in order to access RIS calibrations for those platforms. Environmental objects whose distances gerbils might be expected to compute, though, are characterized by both distinctive local features and unique spatial contexts. In Experiment 3, we assessed the ability of gerbils to use spatial context and local feature information when it is presented together. In addition, we tested the effects of throwing these two types of information into conflict such that the gerbils were presented with probe platforms in mismatched spatial contexts and local features relative to those seen during training.

\section{Method}

Subjects. Eight male gerbils ranging in age from 3 to 6 months were the subjects in this experiment. Housing and maintenance were identical to those described for Experiments 1 and 2 .

Apparatus. The same training platforms were used as those described in Experiment 2, and the same two spatial contexts as those described in Experiment 1 were employed.

Training. Preliminary training sessions were the same as those described for Experiments 1 and 2. Following initial training, the gerbils received training in each of the two testing environments every day. In each environment, the gerbils were presented with a series of 10 trials with either the wide or the narrow platform and either the striped or the dotted texture on the landing platform. Whichever size and texture of landing platform was presented in one room, the opposite size and texture was presented in the other room; these training conditions were kept consistent for each animal for the duration of this phase of training. Test environment was varied randomly from trial to trial. Spatial context and local feature information was counterbalanced across animals.

Testing. In the test phase of the experiment, the gerbils were presented with a total of 76 trials. These trials consisted of control trials that were identical to those presented in training (i.e., randomized distances but particular patterns and widths of platforms invariably associated with particular spatial contexts) and two different types of probe trials. In match probe trials, the gerbils were presented with matching local pattern and spatial context information (e.g., if a gerbil had normally been presented with a striped landing platform in Test Room A during training, then it was presented with stripes in Room A for a match probe trial), but a new landing platform of intermediate width was used. In mismatch probe trials, the gerbils were presented with nonmatching local pattern and spatial context information (i.e., if a gerbil had normally been presented with a striped landing platform in Test Room A during training, then it was presented with dots in Room A for a mismatch probe trial). On each day of testing, the gerbils were presented with two match probe trials and two mismatch probe trials interleaved with 15 control trials. Testing was continued until the gerbils had received one of each kind of probe trial at each of four distances $(10$, 16,22 , and $28 \mathrm{~cm}$ ).

Analysis. Trials were videotaped, and the same analysis as that described for Experiments 1 and 2 was conducted.

\section{Results}

Figure 3 shows the mean landing position of gerbils as a function of gap distance for the match condition. For comparison purposes, performance on the control condition is plotted on the same axes. As can be seen in the figure, substitution of the probe platform produced overjumps or underjumps, depending upon whether it was presented in the situation that was normally associated with the wide or narrow training platform (i.e., if the wide platform was expected, the probe platform produced overjumps; if the narrow platform was expected, the probe produced underjumps). Statistical analysis of these results was conducted using a repeated measures ANOVA. Results of the ANOVA showed that there was a significant effect of probe type [control vs. expected narrow vs. expected wide, $F(2,14)=8.17, p<.05]$, but there was no effect of distance $[F(3,21)=2.11, p>.1]$ and no significant probe $\times$ distance interaction $[F(6,42)=2.002$, $p>.05]$. Post hoc comparisons among means (Tukey) revealed significant differences between control and expected narrow $[Q(3,14)=4.04, p<.05]$ and between expected narrow and expected wide conditions $[Q(3,14)=$ $6.19, p<.05]$.

Figure 4 shows the results for the mismatch condition, with the same control trials as shown in Figure 3 for comparison purposes. As is clear from the figure, there were no significant effects of probe type $[F(2,14)=0.216$, $p>.1]$ or distance $[F(3,21)=2.21, p>.1]$, and there was no significant interaction between probe and distance $[F(6,42)=0.804, p>.1]$.

To measure trial-to-trial consistency in performance, we computed a variance measure for individual gerbils, similar to that used in Experiments 1 and 2. For each ger-

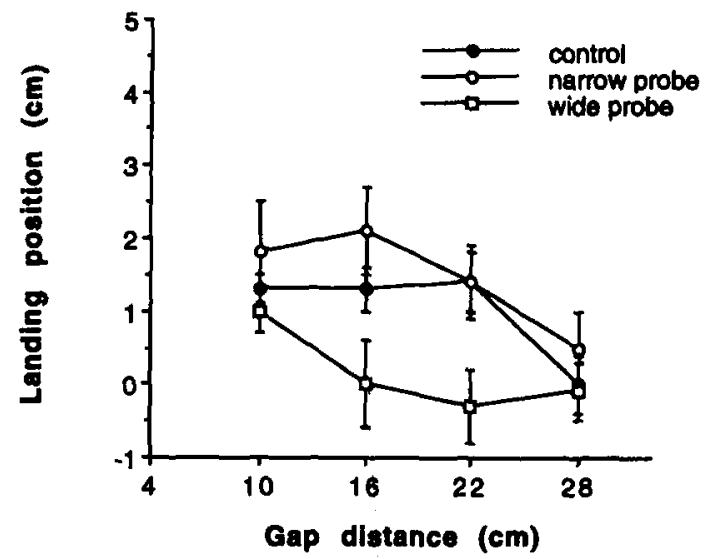

Figure 3. Line graph showing mean landing position as a function of gap distance in the probe trials in the match conditions and the control condition. Solid circles represent performance on control trials. Open circles represent performance on those trials in which the narrow training platform would be expected if the animals were using local features to identify the target. Open squares represent performance on those trials in which the wide training platform would be expected if the animals were using local features to identify the target. Performance is averaged over all animals. Error bars represent standard errors of the mean. 


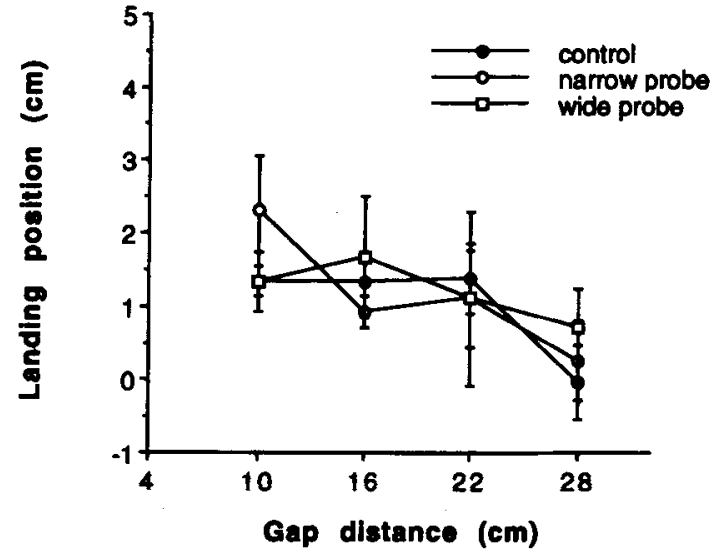

Figure 4. Line graph showing mean landing position as a function of gap distance in the probe trials in the mismatch conditions and the control condition. Solid circles represent performance on control trials. Open circles represent performance on those trials in which the narrow training platform would be expected if the animals were using local features to identify the target. Open squares represent performance on those trials in which the wide training platform would be expected if the animals were using local features to identify the target. Performance is averaged over all animals. Error bars represent standard errors of the mean.

bil, we computed the $S D$ in landing position on control trials and on both the mismatch and the match probe trials. Because average landing positions on the narrowexpected and wide-expected probe trials differed, we computed two separate $S D$ scores for each of the probe types and averaged them. Hence, the data for analysis consisted of three scores for each animal: an error score for the control condition, and one score each for the match and mismatch conditions. These values were analyzed using a repeated measures ANOVA. As shown in Figure 5, there was a significant effect of probe type on error $[F(2,14)=6.86, p<.05]$. Post hoc comparisons among means showed that the only significant effect was an increase in error in the mismatch probe condition relative to the control condition $[Q(3,14)=5.23, p<.05]$.

\section{Discussion}

In contrast to the findings from Experiment 2, the present results suggest that local feature information, although it is not used to identify the targets for the purpose of retrieving RIS calibrations, can influence target recognition when it does not match spatial context. The evidence for this statement comes from a comparison of the outcomes in the match and mismatch conditions. In the former, the congruent spatial context and local feature information provided enough information to retrieve accurate RIS calibrations. In the latter condition, mismatched local feature and spatial context information eliminated the probe effects, suggesting that the gerbils could no longer uniquely identify each of the two platforms for the purpose of retrieving an RIS calibration. In view of the findings in the first two experiments, this outcome is surprising. A straightforward interpretation of the results of Experiments 1 and 2 suggests that gerbils identify objects in this task on the basis of where they are found and not what they look like. If this were true, then the prediction for the mismatch condition in Experiment 3 would be that the gerbils would ignore local features and treat the probe trials in a similar fashion to those presented in Experiment 1, where local features were absent.

As in Experiment 1, we produced a decrease in accuracy by preventing the use of RIS in the mismatch condition. Many previous experiments have shown that gerbils are remarkably resilient to removal of potential sources of distance information. Abolition of binocular cues by lid suture or of loom information, although increasing the use of retinal motion information, has no significant effect on jump accuracy (Ellard et al., 1984), nor does strobe illumination (Long, 1994). Goodale et al. (1991) removed the availability of RIS information on a long-term basis by training gerbils to jump to landing platforms without distinguishing features that were varied in width from trial to trial. Although this manipulation increased reliance on retinal motion, there was no overall decrease in jumping accuracy. Given previous failures to affect accuracy on this task with anything less than large brain lesions (Ellard, Goodale, MacLaren-Scorfield, \& Lawrence, 1986; Ellard \& Ilkov-Moor, 1995), it is all the more remarkable that producing momentary confusion about the identity of the target in Experiment 3 produced a decrease in accuracy. Previously, we have suggested that gerbils are able to weight the value that they place upon separate sources of depth information in this task, depending on prevailing conditions. Removing, or rendering unreliable, one or more sources of depth information has the effect of increasing reliance on other independent sources of information, with no loss of accuracy (Ellard \& Goodale, 1991). In all previous studies, however, gerbils have had a large number of training trials to adapt to changes in the availability or reliability of depth information. The present study sheds new light on this adaptation process by suggesting that such extensive training exposure is probably necessary to effect these kinds of adaptations.

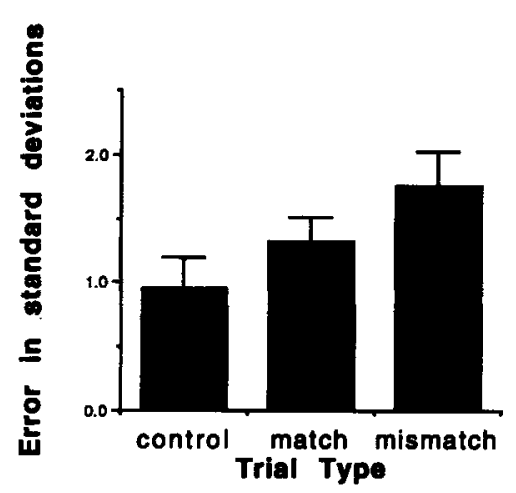

Figure 5. A bar graph comparing errors in performance on the three different types of trial in the experiment. The error score is based on the average of the standard deviation in landing position for each animal, taken across all test distances (see text). Error bars represent standard errors of the mean. 
Although our interpretation of our results is not couched in such terms, it will be apparent that the design of Experiment 3 can be fit to a configural learning paradigm. A simple association learning prediction of the outcome of Experiment 3 would be that since local features were ineffective and spatial context was effective when each type of information was presented alone, their combination in this experiment should yield results that could be predicted on the basis of algebraic summation of these two kinds of effects (Rescorla \& Wagner, 1972). In other words, animals presented with mismatched spatial context and local feature information should respond to the spatial context information alone, much as was the case in Experiment 1. On the other hand, if a configural association (Sutherland \& Rudy, 1989) has been made between the identity of the object and its spatial context and local features, then one would expect the mismatch condition to yield results similar to those presented. When the two types of object attributes were thrown into opposition, the behavior of the animal suggested that it could no longer identify the object. We make no claim that the outcome of this experiment constitutes strong support for or against configural associations, nor was this our intent. To the extent that the experiment can be described in such terms, however, it seems difficult to account for the result in terms of simple associations.

\section{GENERAL DISCUSSION}

When distance is computed using the RIS algorithm, it is necessary to identify the target in order to relate the size of its retinal image to its absolute distance. Our findings suggest that gerbils in such a task are able to identify targets on the basis of their spatial location but not on the basis of clearly distinguishing local features. In studies of discrimination learning (see review by Dean, 1990), even rodents with poorer spatial resolution than gerbils are able to discriminate objects that differ in ways similar to those in our experiments. This suggests that the failure of the gerbils to recognize the targets is task-specific.

In landmark learning studies, Cheng (1986) has shown that, in some circumstances, rats show a remarkable propensity to ignore local feature information in favor of the geometric properties of an environment even when using local feature information could increase accuracy in the task considerably. Indeed, Gallistel (1990) has argued that, in some circumstances, such uses of space by rodents exhibit properties of Fodor's (1983) cognitively impenetrable modules.

In many spatial learning experiments (e.g., Brodbeck, 1994; Collett, Cartwright, \& Smith, 1986), the aspect of local feature information that is of interest was whether or not such information can be used to help an animal define its position in space relative to a target. In a purely formal sense, our experiment tested the same capacity. Local features contributed to the identification of a target, and, once identified, computations of the distance between the target and the animal could take place. However, in our experiments, local feature information, although not sufficient to define an object, appears to have interacted with global spatial information, impeding object identification to the extent that such information was disregarded. Our findings, like those of Cheng (1986) with rats, suggest the primacy of spatial information in object recognition and, like those of Brodbeck (1994) with chickadees, suggest that local feature information can sometimes exert a subtle, modulatory influence on object recognition.

We argue, on these and other grounds (Ellard \& Carey, 1996; Ellard \& Chapman, 1991), that the explanation for these kinds of effects may lie in the organization of the rodent visual system. Rodent vision is afoveal; there is little regional specialization at the retinal level or at any higher level (see Goodale \& Carey, 1990). This type of organization, although quite suitable for the varieties of visuospatial behavior at which rodents excel, is less suited to the analysis of the local features of objects. Given this, we suggest that, when possible, rodents solve object discrimination problems using spatial strategies. In our task, even when local feature information was provided, it was not used. When local feature information conflicted with spatial context data, it influenced the recognition process; however, in this case, one could make the argument that the local features themselves formed a part of the spatial context. After all, stripped of all local features, spatial contexts themselves cannot be differentiated.

There are two main conclusions that can be drawn from these experiments. First, the results suggest that gerbils do not discriminate between objects on the basis of clearly visible differences in local features when the purpose of the object discrimination is to retrieve a correct image size calibration in order to compute a distance. This counterintuitive result suggests that tests of object recognition must take into account the objective of the recognition process. Second, and more speculatively, the results provide evidence in support of our notion that rodent vision is dominated by visuospatial abilities to the extent that these abilities are brought to bear on object recognition problems, even at the expense of clearly visible local features.

\section{REFERENCES}

Baker, A. G., \& Emerson, V. F. (1983). Grating acuity of the Mongolian gerbil. Behavioural Brain Research, 8, 195-209.

BrodBeck, D. R. (1994). Memory for spatial and local cues: A comparison of a storing and a nonstoring species. Animal Learning \& Behavior, 22, 119-133.

Cheng, K. (1986). A purely geometric module in the rat's spatial representation. Cognition, 23, 149-178.

Collett, T. S., Cartwright, B. A., \& Smith, B. A. (1986). Landmark learning and visuo-spatial memories in gerbils. Journal of Comparative Physiology A, 171, 807-815.

COLlETt, T. S., \& HARKNESS, L. (1981). Depth vision in animals. In D. J. Ingle, M. A. Goodale, \& R. J. W. Mansfield (Eds.), The analysis of visual behavior (pp. 111-176). Cambridge, MA: MIT Press.

DEAN, P. (1990). Sensory cortex: Visual perceptual functions. In B. Kolb \& R. C. Tees (Eds.), The cerebral cortex of the rat (pp. 275-308). Cambridge, MA: MIT Press.

EllakD, C. G., \& CAREY, D. P. (1996). Two cortical visual systems in rodents? Manuscript in preparation.

Ellakd, C. G., \& Chapman, D. G. (1991). The effects of posterior cor- 
tical lesions on responses to visual threats in the Mongolian gerbil (Meriones unguiculatus). Behavioural Brain Research, 44, 163-167.

Ellard, C. G., Chapman, D. G., \& Cameron, K. A. (1991). Calibration of retinal image size with distance in the Mongolian gerbil: Rapid adjustment of calibrations in different contexts. Perception \& Psychophysics, 49, 38-42.

Ellard, C. G., \& Goodale, M. A. (1991). Computation of absolute distance in the Mongolian gerbil (Meriones unguiculatus): Depth algorithms and neural substrates. In M. A. Arbib \& J.-P. Ewert (Eds.), Visual structures and integrated functions (pp. 205-219). Berlin: Springer-Verlag.

Ellard, C. G., Goodale, M. A., Maclaren-Scorfield, D., \& LaWRENCE, C. (1986). Visual cortical lesions abolish the use of motion parallax in the Mongolian gerbil. Experimental Brain Research, 64 599-602.

Ellard, C. G., Goodale, M. A., \& Timney, B. (1984). Distance estimation in the Mongolian gerbil: The role of dynamic depth cues. Behavioural Brain Research, 14, 29-39.

Ellard, C. G., \& IlKov-MoOR, S. (1995). The effects of lesions of anteromedial cortex on a ballistic visuomotor task in the Mongolian gerbil (Meriones unguiculatus). Behavioural Brain Research, 68, 53-56.

Epstein, W., \& Baratz, S. S. (1964). Relative size in isolation as a stimulus for relative perceived distance. Journal of Experimental Psychology, 67, 507-513.

FoDor, J. A. (1983). The modularity of mind. Cambridge, MA: MIT Press.
Gallistel, C. R. (1990). The organization of learning. Cambridge, MA: MIT Press.

Goodale, M. A., \& CAREY, D. P. (1990). The role of cerebral cortex in visuomotor control. In B. Kolb \& R. C. Tees (Eds.), The cerebral cortex of the rat (pp. 309-340). Cambridge, MA: MIT Press.

Goodale, M. A., Ellard, C. G., \& Booth, L. (1990). The role of image size and retinal motion in the computation of absolute distance by the Mongolian gerbil (Meriones unguiculatus). Vision Research, 30, 399-413.

INGLE, D. J. (1981). New methods for analysis of vision in the gerbil. Behavioural Brain Research, 3, 151-174.

LONG, A. (1994). The effect of strobe illumination on jumping performance in the Mongolian gerbil. Unpublished honours thesis, University of Waterloo, Waterloo, ON, Canada.

Rescorla, R. A., \& WAGNER, A. R. (1972). A theory of Pavlovian conditioning: Variations in the effectiveness of reinforcement and nonreinforcement. In A. H. Black \& W. F. Prokasy (Eds.), Classical conditioning: II. Current research and theory (pp. 64-99). New York: Appleton-Century-Crofts.

SUTHERLAND, R. J., \& RUDY, J. W. (1989). Configural association theory: The role of the hippocampal formation in learning, memory, and amnesia. Psychobiology, 17, 129-144.

(Manuscript received February 27, 1995; revision accepted for publication May 13, 1995.) 